\title{
Bilateral Paraneoplastic Vitritis: A Case Report
}

\author{
Ezgi Karatas Yigitaslan, ${ }^{1}$ (1) Eyup Sabri Ucan, ${ }^{2}$ () Nurullah Akkoc, ${ }^{3}$ () Ali Osman Saatci ${ }^{1}$ \\ 1Department of Ophthalmology, Dokuz Eylul University, Izmir, Turkey \\ ${ }^{2}$ Department of Pulmonary Medicine, Dokuz Eylul University, Izmir, Turkey \\ ${ }^{3}$ Department of Rheumatology,Celal Bayar University, Manisa, Turkey
}

\begin{abstract}
This report describes the case of a patient who presented with bilateral vitritis that led to a diagnosis of small-cell lung cancer (SCLC). A 70-year-old man was examined due to bilateral gradual visual deterioration. Symmetrical vitritis was observed in both eyes with no evidence of other fundus pathology. The opthalmological diagnosis was bilateral paraneoplastic vitritis. The patient was an active smoker and had a short history of weight loss. A systemic evaluation resulted in a diagnosis of SCLC. The appropriate cancer treatment was provided, as well as a short-term oral steroid. The vitritis responded well to steroid treatment. Ocular manifestations can be an early sign of malignancy, and ophthalmologists should be aware of this possibility.
\end{abstract}

Keywords: Paraneoplastic syndrome, small-cell lung cancer, uveitis, vitritis

\section{Introduction}

Uveitis is a major cause of blindness all over the world (I). A multidisciplinary approach is often necessary to investigate the etiology and tailor the treatment strategy accordingly. There is an established association between uveitis, underlying malignancy, and the immune response to tumors, including pseudouveitis or other forms of masquerade syndrome (2). Abnormal immune reactivity triggered by a malignancy can also cause a rare disorder known as paraneoplastic syndrome (PNS). The signs of disease are not actually related to the metastatic spread or direct invasion of a tumor, rather the mechanism is the production of antibodies against the tumor antigens and the cross-reaction of these antibodies to the normal host tissue (3). PNS is most commonly seen in middle-aged and elderly individuals, and is most often associated with lung, ovarian, lymphatic, or breast cancer (4). The symptoms are usually related to the endocrine, neuromuscular, musculoskeletal, cardiovascular, cutaneous, hematological, gastrointestinal, renal, or neurological systems. It has been estimated that PNS may affect up to $8 \%$ of cancer patients (5). Ocular PNS may present as carcinoma-associated retinopathy (CAR), melanoma-associated retinopathy (MAR), bilateral diffuse uveal melanocytic proliferation, optic neuropathy, paraneoplastic vitelliform retinopathy, opsoclonus-myoclonus syndrome, or Lambert-Eaton myasthenic syndrome (6).

The diagnosis of bilateral paraneoplastic vitritis in this case led to the detection of small-cell lung cancer (SCLC).

How to cite this article: E. Karatas Yigitaslan, E.S. Ucan, N. Akkoc, A.O. Saatci. Bilateral Paraneoplastic Vitritis: A Case Report. Beyoglu Eye J 202I; 6(2): $140-144$.

Address for correspondence: Ezgi Karatas Yigitaslan, MD. Dokuz Eylul Universitesi Goz Hastaliklari Anabilim Dali, Izmir, Turkey Phone: +90 5542438342 E-mail: e.karatas.2015@gmail.com

Submitted Date: November 25, 2020 Accepted Date: January 16, 2021 Available Online Date: June 08, 2021

${ }^{\circ}$ Copyright 2021 by Beyoglu Eye Training and Research Hospital - Available online at www.beyoglueye.com OPEN ACCESS This work is licensed under a Creative Commons Attribution-NonCommercial 4.0 International License. 


\section{Case Report}

A 70 -year-old male smoker (I pack a day for 40 years) presented in April 2014 with painless, subacute, bilateral visual decline of I month's duration. His past medical history was unremarkable; however, weight loss of $17 \mathrm{~kg}$ in recent months as well as myalgia and fatigue were noted.

Examination revealed a bilateral best corrected visual acuity of 20/30. There was no color vision loss. The intraocular pressure values were within the normal range in both eyes. While the anterior chamber was quiet, mild to moderate vitritis was observed in both eyes. Despite the mildly hazy view, indirect ophthalmoscopy yielded no change in the posterior segments (Fig. I a-h). A fluorescein angiogram revealed no abnormality (Fig. I e, f).

Optical coherence tomography images displayed normal foveal architecture with a few hyper-reflective dots in the posterior vitreous of both eyes corresponding to the vitreous cells (Fig. I g, h). The initial diagnosis was bilateral intermediate uveitis.

A full systemic and laboratory work-up was performed in collaboration with a rheumatologist and pulmonologist. Lab investigations included a complete blood count; analysis of serum liver enzymes, renal function, electrolytes, inflammatory markers (erythrocyte sedimentation rate, C-reactive protein), angiotensin-converting enzyme, and rheumatological markers (antinuclear antibody, antineutrophil cytoplasmic antibodies, and rheumatoid factor); infection screen- ing (syphilis and tuberculosis); brain magnetic resonance imaging; and chest radiography. Treatment with $40 \mathrm{mg}$ oral prednisolone was initiated once an infectious etiology was excluded. Finally, positron emission tomography (PET-CT) scanning was used to explore the possibility of sarcoidosis or malignancy.

The PET-CT images revealed an increased uptake of 2-(fluorine-18)-fluoro-2-deoxy-D-glucose in area of the hilar and mediastinal lymph nodes (Fig. 2). To determine the nature of the primary tumor, a biopsy was performed during a bronchoscopy. SCLC (T2N3M0) was established the histopathological examination. The patient was then referred to an oncologist for additional treatment.

After 2 weeks of oral steroid therapy, a subsequent examination yielded a visual acuity of 20/20 and very little lingering bilateral haze (Fig. 3). The patient began a course of chemotherapy, and oral steroid treatment was tapered and terminated. The final diagnosis was bilateral paraneoplastic vitritis. No further visual symptoms were reported during follow-up. Unfortunately, the patient passed away in 2019.

\section{Discussion}

SCLC is the most common cancer associated with PNS. PNS can occur in 2 forms: the first is associated with peptide production, such as adrenocorticotropic hormone, amylase, atrial natriuretic peptide, renin, prolactin, or growth hormone. The second form is caused by an antibody-related immune response, such as Lambert-Eaton myasthenic syn-
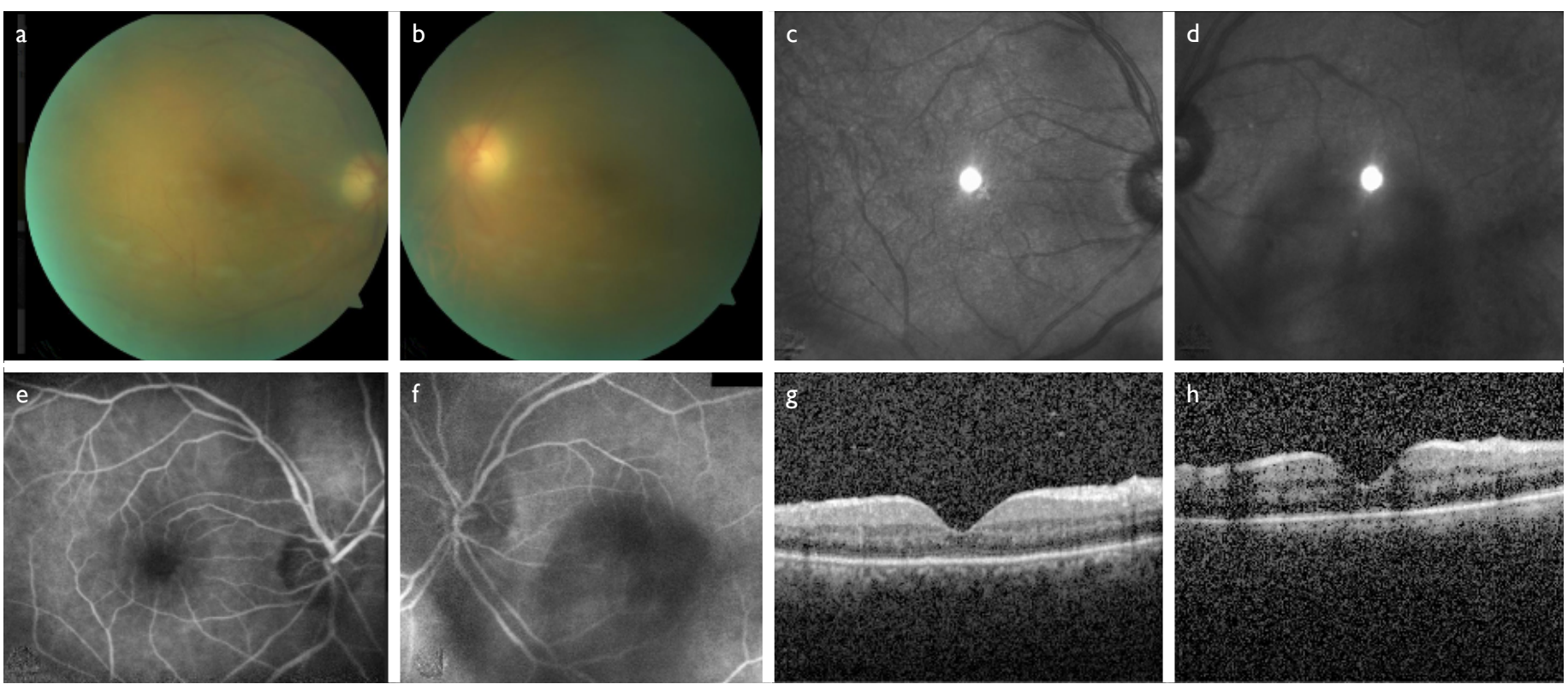

Figure I. Color fundus photograph of the (a) right and (b) left eye illustrating the hazy view of the posterior poles. Please note that both optic discs were slightly obscured due to vitritis, though it was more marked in the left eye. (c-d) Infrared images depicting the vitritis-related blocking effect, which was also more prominent in the left eye. (e-f) Late venous fluorescein angiographic images of both eyes demonstrating no leakage or any sign of vasculitis. The masking effect of the vitritis was more significant in the left eye. (g-h) Spectral domain optical coherence tomography images showing that the macular anatomy was well-preserved and that the inflammatory vitreous cells were visualized as hyper-reflective dots. 

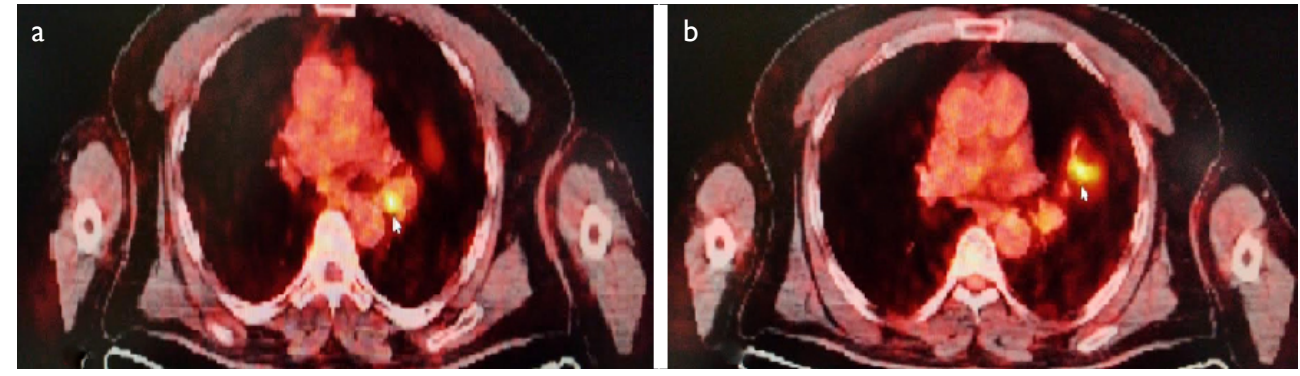

Figure 2. (a) A positron emission tomography scan demonstrating increased uptake of 2-(fluorine-18)-fluoro-2-deoxy-D-glucose in the area of the mediastinal lymph nodes (arrow) and (b) the tumor.
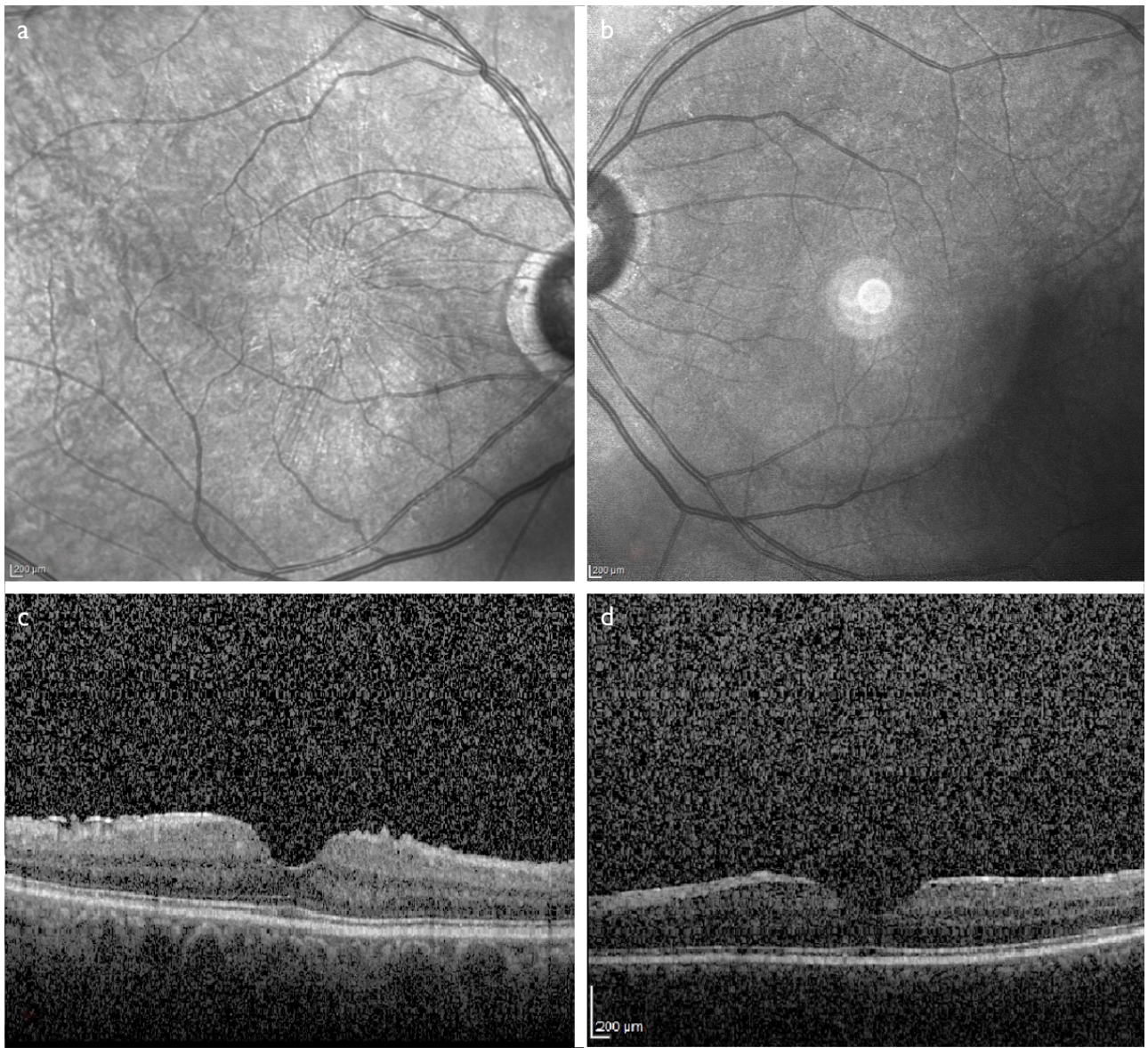

Figure 3. Infrared images of the (a) right and (b) left eye demonstrating improved fundus appearance. Spectral domain optical coherence tomography images of the (c) right eye and the (d) left eye illustrating that the vitritis responded well to the treatment and the lack of hyper-reflective cells.

drome, encephalomyelitis, neuronopathy, cerebellar degeneration, retinopathy, or opsoclonus- myoclonus syndrome (7). The emergence of PNS can predate a diagnosis of cancer by months to years, especially in cases presenting with neurological signs. Life expectancy following the presentation of PNS is 9 to II months; however, the median length of survival can be prolonged to 24 months (8).

A visual disturbance may be caused by uveitis, retinitis, optic neuropathy, or other neurological changes. CAR and MAR are among the most frequently seen clinical types of
PNS. However, intraocular inflammation may present as panuveitis or optic neuropathy (6-9). Characteristic clinical features of CAR can be summarized as a progressive loss of vision, photopsia, ring scotoma or concentric visual field defects, and abnormal electroretinography (ERG) findings. Serum autoantibodies specific to retinal antigens may be present in cases of CAR, but the necessary laboratory tests may not always be available (I0). At least 10 antibodies have been associated with the pathogenesis of CAR (II). Careful assessment of the clinical findings is extremely important, as 
they may vary from no obvious ophthalmological signs to a granulomatous type of uveitis.

Donosa et al. (12) observed that an immunological reaction to the retinal S-antigen might cause extensive ocular inflammation and photoreceptor damage. Autoantibody heterogeneity may be an explanation for the variation and complexity of the clinical symptoms in these patients.

Retinopathy can occur prior to, concurrent with, or after a cancer diagnosis. Hoogewoud et al. (13) reported that CAR symptoms of posterior segment inflammation with a visual field loss and reduced ERG amplitudes at the initial presentation of 4 patients prompted investigation and the ultimate discovery of previously unknown malignancies. Two of the patients were diagnosed with SCLC, I with prostate carcinoma, and I with uterine sarcoma. Saatci et al. (14) described the case of a patient who was diagnosed with a neuroendocrine tumor before the diagnosis of CAR that included bilateral peripheric concentric visual field narrowing and depressed scotopic responses observed with ERG.

Cassell et al. (9) studied 2 patients with bilateral visual loss, vitritis, and pale, swollen optic discs who were positive for antibodies against collapsin response-mediator protein 5 (anti-CRMP-5) and later diagnosed with SCLC. Both patients presented with moderate vitritis, a quiet anterior chamber, and normal macular appearance. The optic discs were pale and swollen, with disc leakage noted on a fluorescein angiogram. The presence of CRMP-5 in the retinal ganglion cells, nerve fiber layer, and photoceptors may explain the inflammation (15).

Cross at al. (I5) studied 16 patients with a paraneoplastic entity of combined optic neuritis and retinitis accompanied by inflammatory vitreous cells. Eleven of the 16 patients had SCLC and 6 had vitritis. Eleven patients had multiple positive autoantibody markers of an immune response initiated by small-cell carcinoma (ANNA-I, $\mathrm{Ca}^{2}$ channel [P/Q and N types], amphiphysin, GAD65) as well as anti-CRMP-5 antibodies. The authors concluded that paraneoplastic inflammatory uveitis was a unique and rare disorder.

Recently, McGrath et al. (6) provided the results of the largest known study of cytologically proven granulomatous vitritis in association with systemic malignancies. The average age at presentation among 8 patients was 70 years (range: $5 \mathrm{I}-89$ years). Six patients had bilateral eye involvement, while the remaining 2 had unilateral eye involvement. Systemic malignancy was diagnosed in 3 of the 8 cases only after the eye involvement. All 5 patients with a prior diagnosis of cancer had a major relapse, metastasis, or both at the time of the appearance of ocular symptoms. Four of these malignancies were either leukemia or lymphoma, 3 cases were classified as various carcinomas, and there was I case of pheochromocytoma. The authors suggested that granulomatosis inflammation may arise when the host immune system fails to completely clear the antigenic stimuli. This pattern of chronic inflammation consisted of a microscopic aggregation of macrophages that were transformed into epithelioid cells surrounded by a collar of mononuclear leukocytes, principally lymphocytes.

Our patient was examined following bilateral subacute visual deterioration together with a complaint of floaters. Although moderate vitritis was observed, the anterior chambers and the fundus of both eyes were surprisingly quiet. In addition, a fluorescein angiogram revealed no pathological findings. A systemic work-up established the diagnosis of SCLC. This case clearly illustrates the importance of a careful clinical evaluation of patients with posterior segment inflammation, particularly the elderly, even when it may appear to be simply vitritis, in order to explore the role or possible presence of a malignancy.

\section{Disclosures}

Informed consent: Written informed consent was obtained from the patient for the publication of the case report and the accompanying images.

Peer-review: Externally peer-reviewed.

Conflict of Interest: None declared.

Authorship Contributions: Involved in design and conduct of the study (EKY); preparation and review of the study (AOS); data collection (EKY).

\section{References}

I. Bansal R, Gupta V, Gupta A. Current approach in the diagnosis and management of panuveitis. Indian J Ophthalmol 2010;58:45-54. [CrossRef]

2. Lannetti L, Corsi C, lafrate F, Sammartino P, Di Giorgio A, Pezzi PP. Bilateral uveitis with hypopyon as a presenting symptom of metastatic peritoneal carcinomatosis. Eur J Ophthalmol 2010;20:948-51. [CrossRef]

3. Adamus G. Autoantibody targets and their cancer relationship in the pathogenicity of paraneoplastic retinopathy. Autoimmun $\operatorname{Rev} 2009 ; 8: 410-4$. [CrossRef]

4. National Institute of Neurological Disorders and Stroke. Paraneoplastic Syndromes Information Page. Available at: https:// www.ninds.nih.gov/disorders/All-Disorders/ParaneoplasticSyndromes-Information-Page. Accessed August 16, 2020.

5. Pelos of LC, Gerber DE. Paraneoplastic syndromes: an approach to diagnosis and treatment. Mayo Clin Proc 2010;85:838-54.

6. McGrath LA, Mudhar HS, Sheard R, Spiteri-Cornish K, Winder $\mathrm{S}$, Rundle $\mathrm{P}$, et al. Paraneoplastic granulomatous vitritis: elaboration of 8 cases. Ophthalmol Retina 2019;3:589-96. [CrossRef]

7. Gandhi L, Johnson BE. Paraneoplastic syndromes associated with small cell lung cancer. J Natl Compr Canc Netw 2006;4:631-8. [CrossRef]

8. Maddison P, Newsom-Davis J, Mills KR, Souhami RL. Favourable 
prognosis in Lambert-Eaton myasthenic syndrome and smallcell lung carcinoma. Lancet 1999;353:I I7-8. [CrossRef]

9. Casswell EJ, Pringle E, Thuang C, Sanders MD, Graham EM. Clinical and histological features of small cell lung cancer paraneoplastic inflammatory uveitis. Ocul Immunol Inflamm 2016;24:503-7. [CrossRef]

10. Makiyama Y, Kikuchi T, Otani A, Oishi A, Guo C, Nakagawa S, et al. Clinical and immunological characterization of paraneoplastic retinopathy. Invest Ophthalmol Vis Sci 20I 3;54:5424-3 I.

II. Parc CE, Azan E, Bonnel S, Sahel JA, Kaplan J, Thirkill CE. Cone dysfunction as a paraneoplastic syndrome associated with retinal antigens approximating 40 kiloDalton. Ophthalmic Genet 2006;27:57-6I. [CrossRef]
12. Donoso LA, Yamaki K, Merryman CF, Shinohara T, Yue S, Sery TW. Human S-antigen: characterization of uveitopathogenic sites. Curr Eye Res 1988;7:1077-85. [CrossRef]

13. Hoogewoud F, Butori P, Blanche P, Brézin AP. Cancer-associated retinopathy preceding the diagnosis of cancer. BMC Ophthalmol 20।8; 18:285. [CrossRef]

14. Saatci Ali Osman, Ayhan Z, Seymenoglu G, Unlu B. Cancerassociated retinopathy and Its clinical features. Retina-Vitreus 2017;26:69-72.

I5. Cross SA, Salomao DR, Parisi JE, Kryzer TJ, Bradley EA, Mines JA, et al. Paraneoplastic autoimmune optic neuritis with retinitis defined by CRMP-5-lgG. Ann Neurol 2003;54:38-50. [CrossRef] 